\title{
Preliminary Calculations for Minehunter's Genset Foundations
}

\author{
Andrzej Grządziela, Marcin Kluczyk
}

The requirements for the foundations of naval vessel engines are based on classification rules devised by classification societies or/and military standardization rules. A class guideline was published, defining the requirements, acceptance criteria and machinery seating methods. There is also a schedule of basic calculation procedures for cast resin or rubber chocks which facilitates the process of machinery foundation design, preloading force calculation, the establishment of boundary conditions etc. In case of naval vessels, typical procedures do not meet tactical requirements due to potential explosion loads. Since minehunters are mainly deployed at sea, handling active, naval mines, the shock resistance calculation for the entire machinery, including the Genset, is required. The paper presents preliminary MatLab calculation methods which can be used to analyse the type, number and location of rubber or elastomer chocks. The procedure consists of input data such as UNDEX pressure and technical data of the Genset used in the Polish Navy. Calculation results include the analysis of the dynamic interaction between the Genset and the foundation, as well as the damping effect generated by the UNDEX (UNDerwater EXplosion) shock pulse.

\section{KEY WORDS}

$\sim$ Minehunter

$\sim$ Foundation

$\sim$ Shock

$\sim$ Undex

Faculty of Mechanical and Electrical Engineering, Polish Naval Academy, Poland e-mail: a.grzadziela@amw.gdynia.pl

doi: $10.7225 /$ toms.v08n01.006

This work is licensed under (cc) BY

\section{INTRODUCTION}

The foundations of machines and engines on naval ships have been designed in accordance with the regulations of marine classification societies and military standardization. Since each navy has its own test results and naval battle experience, the details of requirements and calculation algorithms are classified or, more frequently than not, confidential documents. The main differences between the machine foundations on commercial vessels and navy ships are the ability to absorb energy from UNDEX (underwater explosion) and hydroacoustic signature reduction by noise and vibration damping. The main recommendations for engine seatings have been defined in the classification rules set by classification societies. The basic requirements for main engine foundations include the following parameters: seating structure thickness, minimum mating surface of cast resin chocks, preloading force exerted on a foundation bolt, tightening torque of foundation bolt, tensile stress depending on the thread root diameter of the foundation bolt on the basis of preloading force, etc. The paper presents a preliminary simulation of a GenSet foundation with introduced new load element i.e. the impact of UNDEX shock pulse.

\section{OBJECT OF RESEARCH, EXTERNAL AND INTERNAL FORCES}

The object analysed in this paper is a Poweras Generating Set MPAP 108, described in Figure 1.

The technical parameters and basic dimensions of this Genset are indicated in Table 1. 


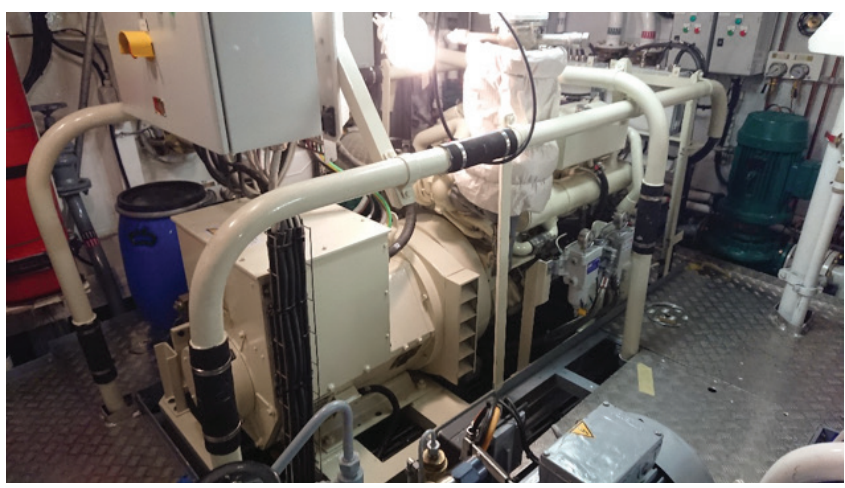

Figure 1.

Object of research - GenSet MPAP - 108 type.

Table 1.

Parameters and dimensions of MAN B\&W 6L16/24 GenSet.

\begin{tabular}{lll} 
Parameter & Value & Unit \\
\hline Type & MPAP 108 & \\
\hline Power & $135(\cos 0.8)$ & $\mathrm{kVa}$ \\
\hline Voltage & $3 \times 400$ & $\mathrm{~V}$ \\
\hline Rotational speed & 1500 & $\mathrm{rpm}$ \\
\hline Engine Power & 122 & $\mathrm{~kW}$ \\
\hline
\end{tabular}

\subsection{External forces}

External forces have been defined as forces originating in the marine environment, unelated to the working processes within the engine. The first external force is slamming, i.e. the impact of a hull bottom in motion on a wavy sea surface. The effect of slamming exposes the high loads exerted on the hull structure and the machinery inside the vessel. The second external force is the impulse of underwater explosion (UNDEX) which affects a sea mine's pulse excitation. The UNDEX in-fluid phenomena consist of the preliminary calculation of the following parameters: resultant pressures, shockwave peak pressure, decay constant, as well as gas bubble parameters for different explosives (Cole, 1948). The shockwave has spherical shape, which, after detonation, expands at a celerity dependant on explosion pressure, from (Keil, 1961):

$C=c\left(1+8,7 \cdot 10^{-4} \cdot p_{0}\right)$

Where: $C$ - is shockwave celerity, $c$ - speed of sound in water, $p_{0}-$ pressure decline on the shock wave [MPa].

The direct propagation of the pressure wave along the vessel's hull was used in the calculations. In the simulation model the distance from the detonation epicentre is four times greater than the diameter of the first gas bubble, which is a realistic scenario for minehunter operations. The model disregarded the effect of pulsation from cavitation and the waves reflected from the sea bed, recognizing their energy value as significantly lower than that of the direct detonation wave. However, it should be noted that further analysis of the impact of both pulsations could be of relevance for calculating the resonance effects of solid structures mounted inside the ship's hull. In empirical formulas, there is maximum pressure $p_{\max }$. Robert Cole (Cole, 1948) described it with the following formula for TNT:

$p_{\text {max }}=523\left(\frac{\sqrt[3]{ } W}{R}\right)^{1,13} \cdot 10^{5}$

where: $W$ - explosive mass TNT, [kg], $R$ - distance from epicentre, [m].

Apart from maximum pressure value, the function describing the performance of pressure in the function of time is also important. Its course is approximated by the following exponential function:

$p(t)=\left[K_{1} \cdot\left(\frac{\sqrt[3]{ } W}{R}\right)\right] e^{-\frac{t-t_{0}}{\theta}}$

where: $t_{0}$ - time calculated from the moment the first pressure wave came into contact with the hull, [ms],K1 - shock wave coefficient, for TNT K1 =52,12, A1 - pressure coefficient, for TNT $\mathrm{A} 1=1,18, \theta$ - time constant [ms], calculated as follows:

$\theta=9,3 \cdot \sqrt[3]{ } W\left[\frac{\sqrt[3]{ } W}{R}\right]^{(-0,22)} \cdot 10^{-5}$

The value of the maximum pressure on the shockwave front is not the only parameter that has the destructive power. The time of its impact should also be taken into account. Both parameters have one relationship called a shock pulse pressure, its value for TNT is calculated as follows (Cole, 1948):

$I=5768 \frac{W^{0,63}}{R^{0,89}}$ 
The equation facilitates the calculation of shock pulse pressure during the first pulsation, provided that the explosion occurs far from both the seabed and the sea surface (the diameter of the first gas bubble is much smaller than depth) (Park et al., 2003). The Boussinesq-Basset force model (added water) was used for simulation calculations of the effect of the shock on the hull (Basset, 1961). The comparison of the result of the hull acceleration simulation model with the result of the UNDEX sea trials is presented in the figure below:

Due to the inevitable use of the Laplace transform to obtain the frequency transform, the Dirac delta impulse response served as the UNDEX impulse model in initial simulations.
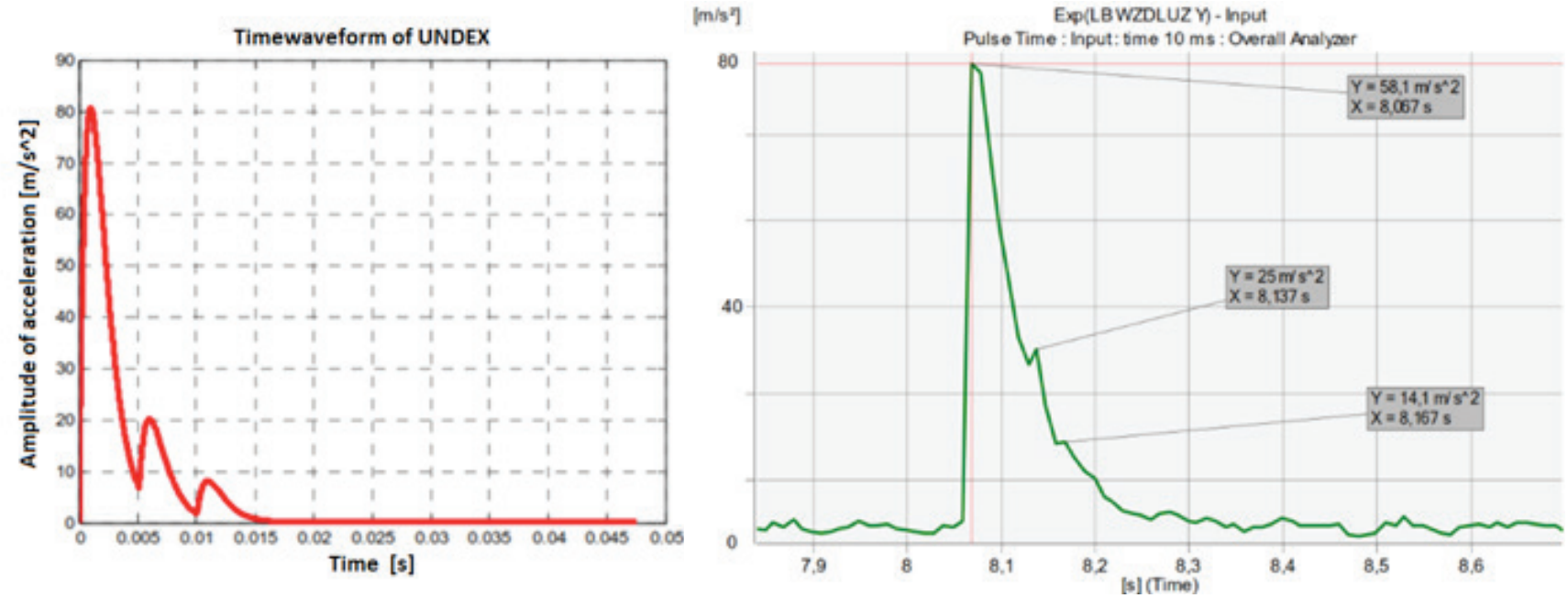

Figure 2 .

Simulation model of impact interaction with 3 successive pulsations of the gas bubble and the magnitude of the detonation effect.

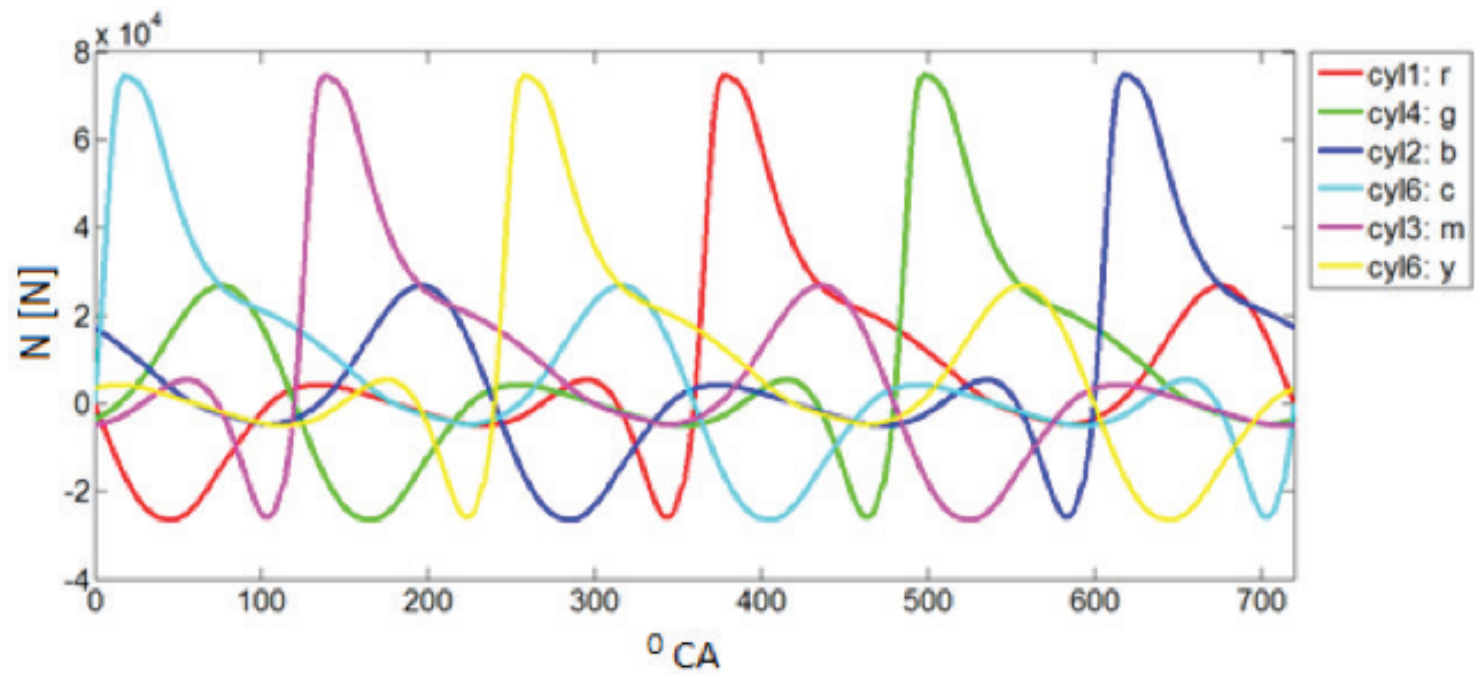

Figure 3.

Fluctuations of tangential forces of individual cylinders of a GenSet, six-cylinder engine. 


\section{INTERNAL FORCES}

Genset's internal forces include inertia forces and gas forces, which is the principle of diesel engine operation. Inertia forces were calculated based on the analysis of the system's crankshaft kinematics and the residual unbalancing of the rotor system in the generator. Other inertia forces were disregarded. After implementing the equations describing the kinematics and dynamics of the crank and piston system in MatLab, defining the geometry and determining the cylinder pressures and the masses performing individual types of movement, the tangent force courses for individual cylinder systems of the 4 stroke, sixcylinder engine were obtained. Figure 3 illustrates the waveforms of individual tangential forces when all cylinders have identical combustion pressures for $90 \%$ of effective engine power (Kluczyk \& Grządziela, 2015). The residual forces generated by generator rotor unbalancing have a very small energy effect but were not disregarded in the calculations due to their potential resonance effect.

The simulation of gas forces was performed as a spline interpolation of four engine work strokes (Kluczyk, Grządziela \& Batur, 2016). During the intake stroke, the cylinder pressure $F_{g}$ remains constant and can be defined as:

$F_{g}=\frac{\Pi D^{2}}{4}\left(p_{d}-p_{0}\right)$

In the next compression, pressure increase can be described by the following equation:

$F_{g}=\frac{\Pi D^{2}}{4}\left\{p_{d}\left[\frac{2 \varepsilon}{(\varepsilon-1)\left(1-\cos \varphi+0,5 \lambda \sin ^{2} \varphi\right)+2}\right]^{n_{1}}\right\}$

In the combustion stroke, the intra-cylinder pressure can approximately be described as:

$F_{g}=\frac{\Pi D^{2}}{4}\left\{p_{d} \varphi_{c} \varepsilon^{n_{1}}\left[\frac{2 \rho}{(\varepsilon-1)\left(1-\cos \varphi+0,5 \lambda \sin ^{2} \varphi\right)+2}\right]^{n_{2}}\right\}$

And finally, the exhaust stroke was described with the following formula:
$F_{g}=\frac{\Pi D^{2}}{4}\left(p_{w}-p_{0}\right)$

where: $p_{0}$ - is atmospheric pressure, $p_{w}$ - outlet gas pressure, $p_{d}$ - air boost (turbo) pressure, $D$ - piston diameter, $\varepsilon$ - compression ratio, $\rho$ - volume increase ratio, $\varphi_{c}$ - pressure increase ratio, $n_{1}$ polytropic compression exponent, $n_{2}$ - polytropic expansion exponent.

The simulation of Time Waveform and its FFT spectrum of acceleration on the Genset engine foundation are shown in Figure 4.

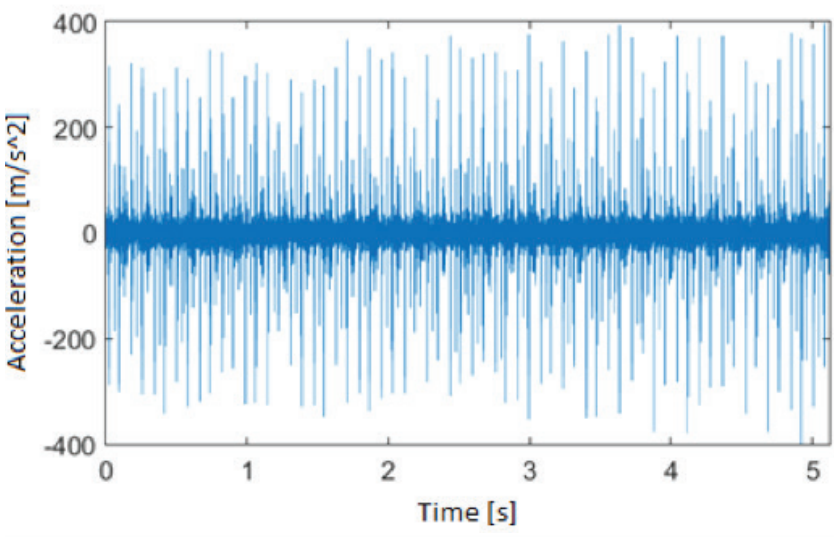

Figure 4.

Simulation of the time course of engine's vibration acceleration, vertical axis.

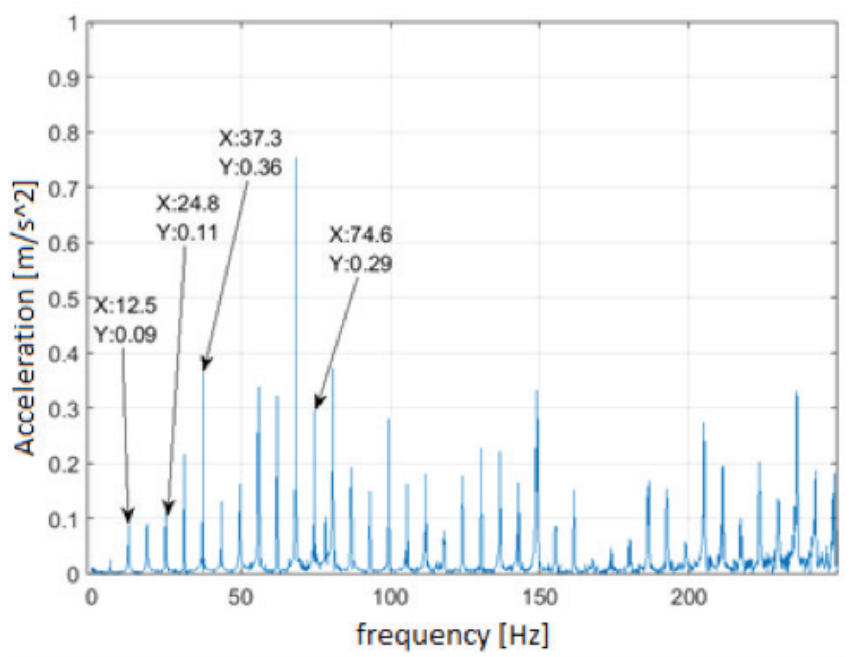

Figure 5.

Simulation of the FFT spectrum of engine's vibration acceleration, vertical axis. 
Finally, the redundant generator unbalancing model was applied. Taking into account standardization rules and electric power, it is assumed the harmonic excitation of the rotor can be described as follows:

$F_{R M}=m_{U B} \sin \omega$

However, although preliminary UNDEX resistance tests do not require engine and generator operation, dynamic models of internal and external loads would be useful for further research. A real operational scenario needs to be set up, with the engine and the generator working during an UNDEX detonation.

\section{MATHEMATICAL AND PHYSICAL MODEL}

Since the supporting floor - hull structure is sufficiently flexible, a two-level model is a sufficiently accurate representation. Figure 6 illustrates the basic forces acting on Genset's mass with respect to time and displacements. Due to the fact that the ship's hull is submerged in sea water and taking into account the acceleration effect of the UNDEX pulse, the sum of the hull's mass and the added water mass was analysed for simulation purposes.

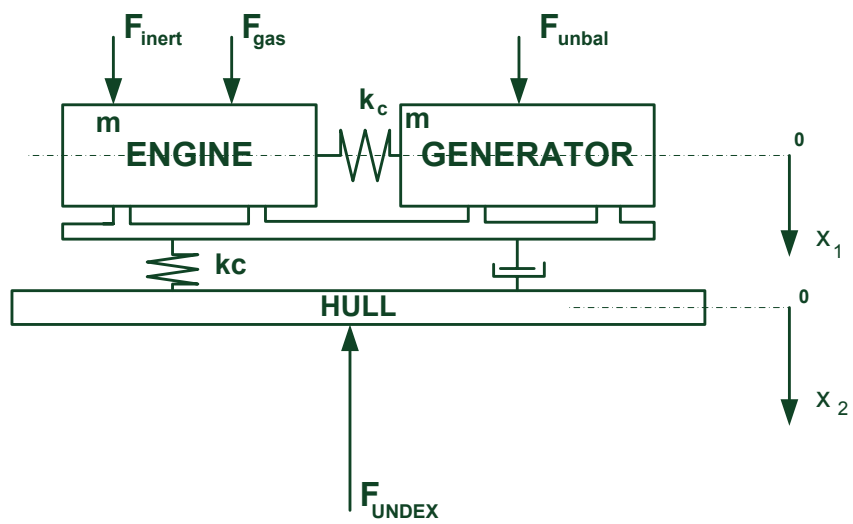

Figure 6.

Scheme of forces acting on Genset

The proposed design of washers assumes a two-stage sandwich-type system, called system 2, opposite to homogeneous system washers - system 1. In system 2, the first, upper washer layer has to withstand static deflection from Genset's mass and the dissipated energy from the dynamic interactions of the inertia forces of the generator and the engine, as well as the engine's gaseous forces and sea wave forces (Tappu, Sen and Lele, 2013). The lower layer has a different physical performance and acts as UNDEX pulse suppressor. The detonation wave affecting the ship's hull was anticipated to transfer its energy to Genset before hull displacement, due to the considerable inertia of the hull and the mass of accompanying water. Task formulated in this manner allows for the application of Newton's second law, as follows:

$F_{n}=F_{g}-(c x-k x)=\left(m \frac{d^{2} x}{d t^{2}}\right)-\left(c \frac{d x}{d t}+k x\right)$

Rewritten (11) to obtain a second order linear differential equation

$\frac{d^{2} x}{d t^{2}}+\frac{c}{m} \cdot \frac{d x}{d t}+\frac{k}{m}=\frac{F_{n}}{m}$

where $x$ is displacement, $c$ is damping resistance and $k$ is the spring constant.

The homogeneous form of (12) can be described as:

$$
\frac{d^{2} x}{d t^{2}}+\frac{c}{m} \cdot \frac{d x}{d t}+\frac{k}{m}=0
$$

and the general solution to (12) is:

$x(t)=x_{H S}(t)+x_{P S}(t)$

where $x_{H S}(t)$ is the general solution to (13), i.e. homogeneous solution, and $x_{p s}(t)$ is the particular solution which depends on $F_{n}$ forms, [Haynes]. Although homogeneous solutions can have three forms of response, only two are of interest in this case, i.e.:

$x_{H S}(t)=C_{1} \cdot e^{a t}+C_{2} \cdot e^{b t}$

$x_{H S}(t)=C_{1} \cdot e^{a t}+C_{2} \cdot t \cdot e^{b t}$

Furthermore, the system is overdamped in the first form and critically damped in the next form. This is to be expected in a proper foundation. The homogeneous solution in this form has constants which are complex conjugates in real solutions. In case of a homogeneous solution (13), they depend on the roots of the characteristic equation:

$s^{2}+\frac{c}{m} s+\frac{k}{m}=0$ 
Eq. (15) illustrates an overdamped system; it has real and distinct roots, i.e. roots $a$ and b. Eq.(16) has the reiterated root $a$ and the system is critically damped.

The system has response with (t) as input and the impulse response denoted as $h(t)$ (Tappu, Sen \& Lele, 2013). The Laplace transform of the system output response - eq. (12) is presented as follows:

$$
X(s)=\frac{F_{n}(s)}{s^{2}+\frac{c}{m} s+\frac{k}{m}}
$$

Where $F_{n}(s)$ is system input. Impulse response $h(t)$ can than be defined as:

$$
h(t)=L^{-1}\left(\frac{1}{s^{2}+\frac{c}{m} s+\frac{k}{m}}\right)
$$

The system transfer function is defined as

$$
\frac{X(s)}{F_{n}(s)}=\frac{1}{s^{2}+\frac{c}{m} s+\frac{k}{m}}
$$

If the input forcing function $F(t)$ is presumed to consist of several pulses, as shown in Figure 2, function $F(t)$ will take the following form:

$$
F(t)=B_{1} \cos \omega t+B_{2} \sin \omega t=C \cdot \cos (\omega t+\varphi)
$$

If time $t$ is presumed to tend to infinity, the general solution of the equation takes the form of a steady state solution and tends to the particular solution ( $t$ ). If $(i \omega)$ is substituted with $s$ in (20), it obtains the following frequency response:

$$
F(\omega)=\left(\frac{1}{i \cdot \frac{c}{m} \omega+\left(\frac{k}{m}-\omega^{2}\right)}\right.
$$

For further analysis, the following simplifications are assumed:

- the system has equivalent stiffness and damping constants, - stiffness and damping were introduced as a flexible coupling connecting the engine and the generator,

- the arrangement of shock absorbers is symmetrical,

- there is only one shock impulse which acts vertically on the center of gravity of the Genset system.

\section{SIMULATION RESULTS}

Due to Genset's constant rotational speed, resonant frequencies were defined as the natural frequency of the overall system and its elements, calculated numerically, as the frequency equal to the rotational speed of the diesel engine - generator, the rotating system. The operational scenario facilitates the calculation of single pulse force of $5000 \mathrm{kN}$ in the duration of $0.005 \mathrm{sec}$, assuming the Dirac impulse shape. The ensuing pulses are disregarded due to the effect of a huge explosive charge and the shallow water along the Baltic coastline.

The result of the numerical calculation of the natural frequencies of the bending vibration in the vertical axis of Gensets' foundation system gave $4.45 \mathrm{~Hz}$ and $3,87 \mathrm{~Hz}$ (respectively for engine and generator), with the first harmonic of the rotational speed of the Genset rotor being $15 \mathrm{~Hz}$. Simulations were made for two different shock absorption systems - system 1 and system 2, with the first consisting of 10 homogenous shock absorbers and the second having the same number of shock absorbers, but a sandwich-type construction. Shock absorbers used in system 2 were constructed as a sandwich elastomeric element, with two layers of different hardness.

Figure 7 shows simulation results for shock absorption system No. 1 and Figure 8 results for system 2. Both simulations were carried out for non-operational Genset, as is the case during sea acceptance tests with UNDEX.

The results indicate a much smaller displacement of both spectral components in system 2. Figure 9 shows the time waveform amplitude of displacement for both systems. It should be noted that both systems are underdamped, although the damping effects are completely different. The solution in system 1 has higher amplitude value and greater number of cycles. System 2 is far from critical damping, however, the use of two layers of the damper sandwich construction, with different hardness, reduced amplitude two times, simultaneously reducing the number of oscillation cycles. The result was obtained for the non-linear characteristics of stiffness and damping of the damper in system 2. Nonlinearity is the effect of bottom layer geometry, which takes prismatic form. In the near future, dampers will be tested further using similar construction and different physical properties of these layers. 


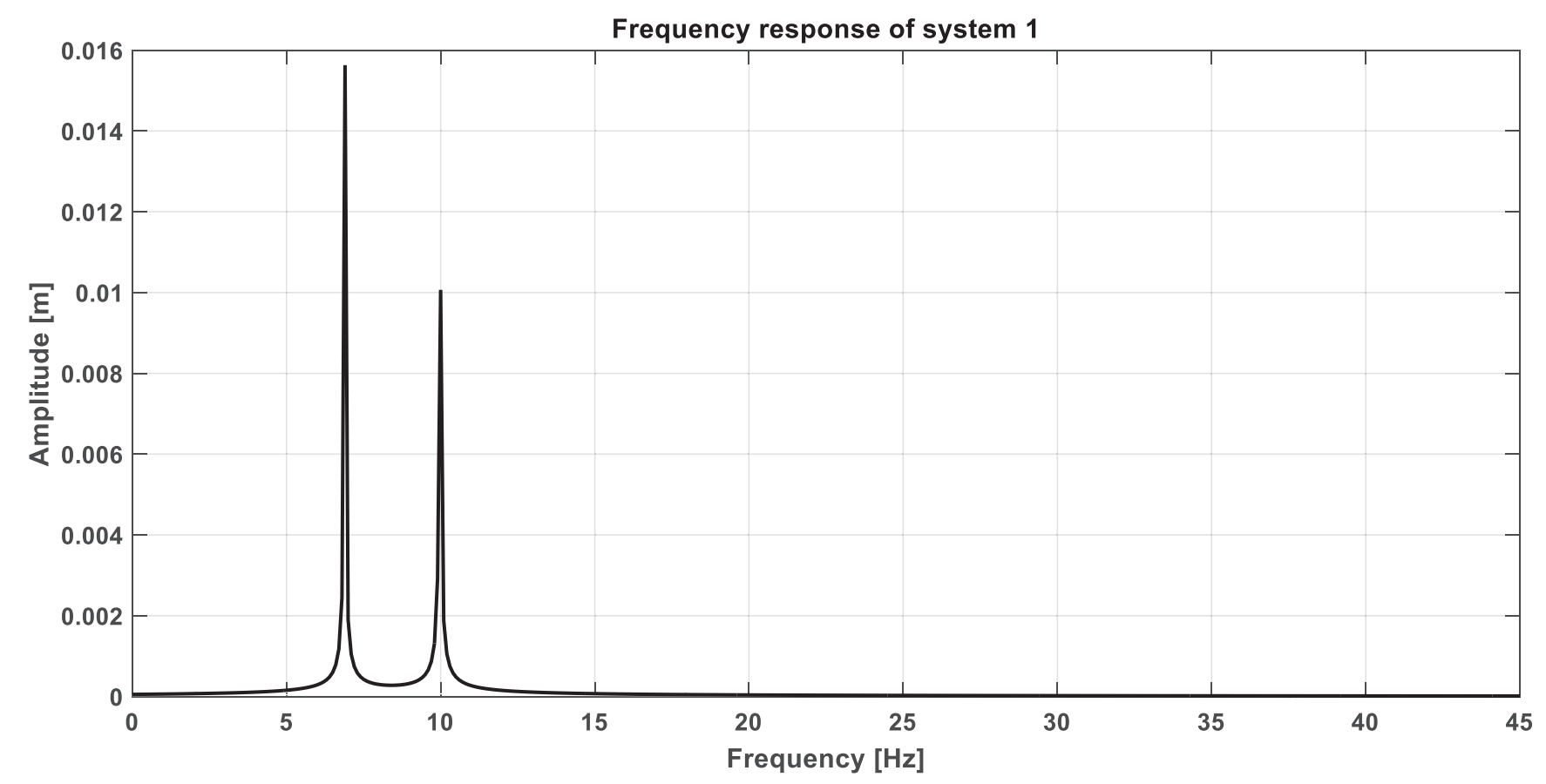

Figure 7.

Simulation spectra of frequency response for system No. 1.

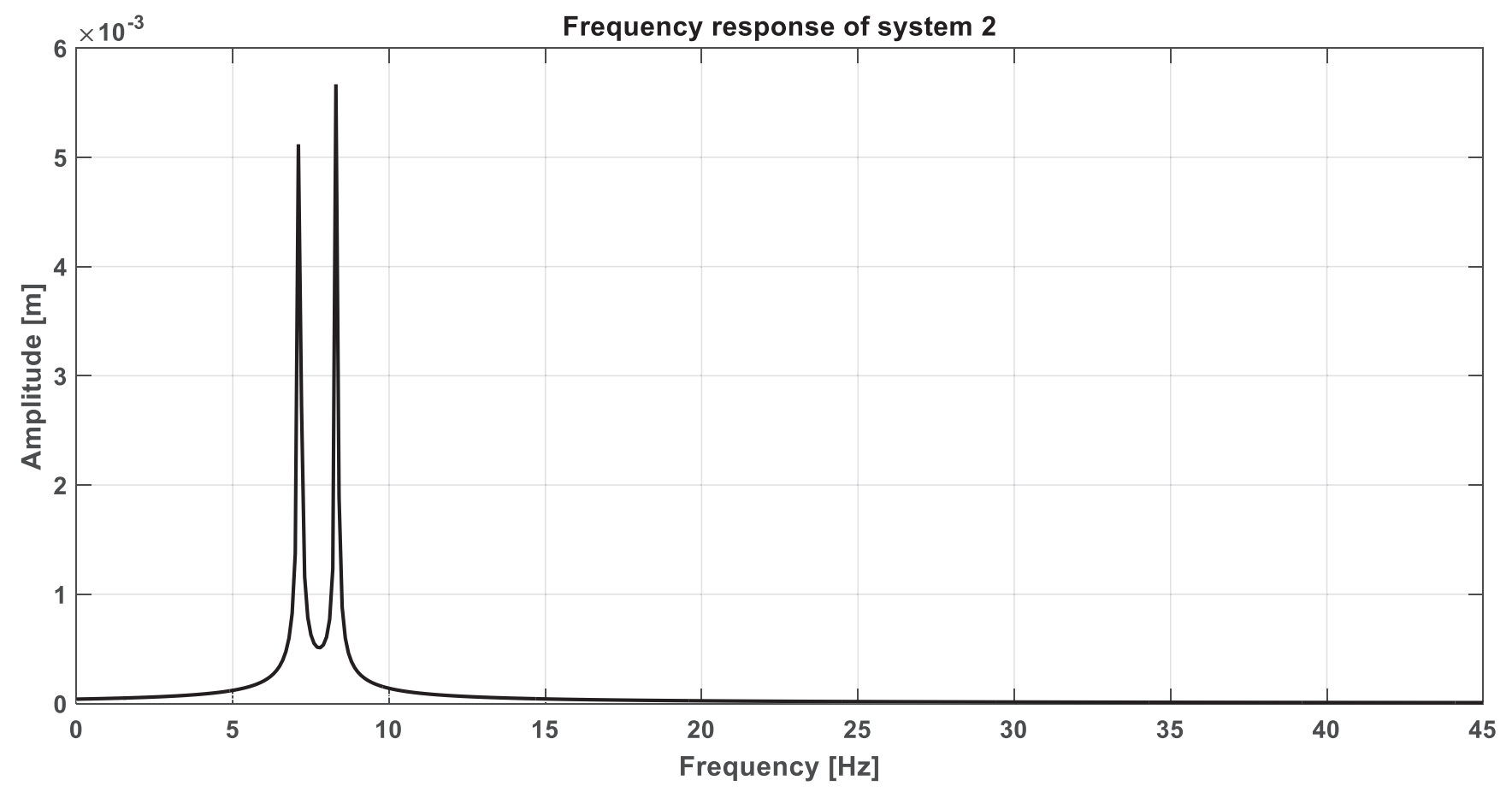

Figure 8.

Simulation spectra of frequency response for system No. 2 . 


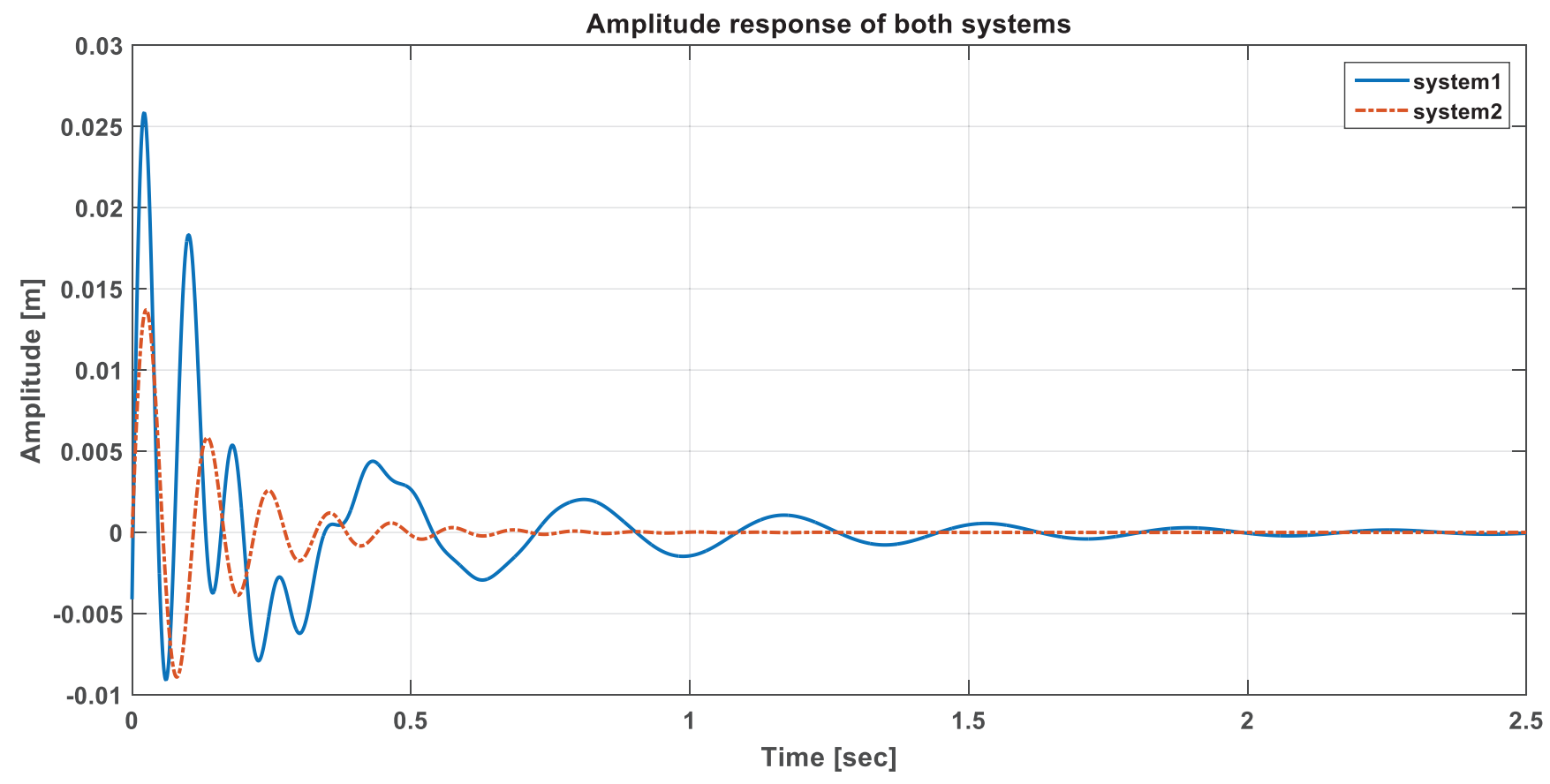

Figure 9.

Simulation of time waveform of displacement for both systems.

The purpose of these tests will be the performance of passive experiments on a small shock machine to determine the damping and stiffness values at high-speed changing load. Genset operating conditions on minehunters require further simulations with both harmonic and pulse loads.

\section{CONCLUSIONS}

The presented research outcomes are the result of the first work package, as part of the project researching the issue of shock resistant foundation of naval machinery. The preliminary tests with a simulated load in the form of Dirac's pulse were carried out on the current package. The next step is the calculation of the unambiguous Laplace transform for the model presented in Figure 2 and the simulation of the response of both systems under simultaneous harmonic and pulse loads.

The simplifications adopted in the paper, including the empirical determination of damper damping and stiffness values, will be developed in the next work package as constructionmaterial models.

The obtained results are satisfactory and, as expected, correspond to the value of a real system's response.

\section{REFERENCES}

Basset, A.B., 1961. Treatise on hydrodynamics, Cambridge: Deighton, Bell and Co., Chapter 22.

Cole, R.H., 1948. Underwater explosions. Available at: https://dx.doi.org/10.5962/bhl.title.48411.

Kluczyk, M., Grządziela, A. \& Batur, T., 2016. Diagnostic Model of the Marine Propulsion System. Applied Mechanics and Materials, 817, pp.57-63. Available at: http://dx.doi.org/10.4028/www.scientific.net/amm.817.57.

Park, I.-K. et al., 2003. Measurement of Naval Ship Responses to Underwater Explosion Shock Loadings. Shock and Vibration, 10(5-6), pp.365-377. Available at: https://dx.doi.org/10.1155/2003/803475.

Keil, A.H., 1961. The Response of Ships to Underwater Explosions. Available at: https://dx.doi.org/10.21236/ad0268905.

Kluczyk, M. \& Grządziela, A., 2015. Simulation Model of Four Stroke, Six Cylinder Marine Diesel Engine. Solid State Phenomena, 236, pp.113-118. Available at: http://dx.doi.org/10.4028/www.scientific.net/ssp.236.113.

Tappu A.L., Sen A.K. \& Lele M.M., 2013. Design sensitivity analysis of raft foundation for marine engines and machinery in warships. International Journal of Engineering Research and Applications, pp.1200-1206. 